Polymer Journal, Vol. 9, No. 2, pp 191-194 (1977)

\title{
Reactivity of Isoprene toward Lithium Diethylamide- Diethylamine Complex
}

\author{
Tadashi Narita, * Yoshiaki Nitadori, and Teiji Tsuruta \\ Department of Synthetic Chemistry, Faculty of Engineering, \\ University of Tokyo, 3-1, Hongo 7-chome, Bunkyo-ku, \\ Tokyo 113, Japan.
}

(Received December 7, 1976)

\begin{abstract}
An addition reaction of diethylamine onto isoprene catalyzed by lithium diethylamide was found to produce selectively 1-diethylamino-3-methyl-2-butene. The regioselectivity of this addition reaction is compared to the results with the sodium amide catalyst system. On the basis of kinetic studies, the comparison of the reactivity of isoprene with that of butadiene is discussed in connection with the initiation reaction of these monomers in anionic polymerization. It is also concluded that the activation of lithium diethylamide by diethylamine causes the rapid addition reaction of the amide anion onto diene and styrene, in contrast with the extremely slow initiation reaction in the polymerizations of these monomers with lithium diethylamide as catalyst in polar solvents.
\end{abstract}

KEY WORDS Isoprene / Lithium Diethylamide / Diethylamine / Addition Reaction / Butadiene / 1-Diethylamino-3-methyl-2-butene /

The typical anionic polymerization of dienes and styrene with the use of metal alkyl catalysts has been extensively studied in recent years, and the reactivities of these monomers toward various types of metal alkyl have now been well established. ${ }^{1}$ On the other hand, few studies have been carried out with metal amide systems.

It was previously reported by us that lithium diethylamide did not induce the polymerization of 1,3-butadiene in the presence of diethylamine, but produced selectively an addition product, 1-diethylamino-cis-2-butene, under appropriate reaction conditions. ${ }^{2}$ It was also shown that no reaction takes place between butadiene and lithium diethylamide in the absence of diethylamine in hydrocarbon media. Studies of kinetics and spectroscopy demonstrated that the $1: 2$ complex consisting of lithium diethylamide and diethylamine is the operating species in this addition reaction. ${ }^{2}$ Tait and coworkers, however, have recently reported that the polymerizations of styrene and isoprene initiated by lithium

* Present address: Department of Environmental Technology, Saitama Institute of Technology, 1690 Fusaiji, Okabe, Osato, Saitama 369-02, Japan. diethylamide itself take place in polar solvents such as tetrahydrofuran of 1,2-dimethoxyethane. $^{4-6}$ These polymerization processes were reported to be anionic in nature and the initiation reactions were shown to be relatively slow compared to the chain propagation process.

In order to reconfirm our previous results for the behavior of lithium diethylamide, we report in this paper that isoprene also undergoes a similar addition reaction, but no polymerization reaction with lithium diethylamide in the presence of diethylamine in cyclohexane as solvent. This paper also treats a kinetic study of the reaction of the amide-amine complex with isoprene in comparison with that of butadiene; here the effect of a methyl group on the reactivity of 1,3-dienes is examined. The addition reactions of an alkyl carbanion to dienes have been extensively studied in terms of the initiation stage of anionic polymerizations of dienes. ${ }^{7-9}$ Information on the relative reactivity of butadiene and isoprene toward alkyllithium and lithium amide may be useful for elucidation of the mechanism of anionic polymerization.

Another object of this paper is to compare 
our results for the selective formation of 1dimethylamino-3-methyl-2-butene with those obtained by the use of sodium dimethylamide, ${ }^{10,11}$ since the regioselectivity in an addition reaction with sodium dimethylamide is rather lower than ours.

\section{EXPERIMENTAL PART}

Most of the experiments were carried out under a purified nitrogen atmosphere in order to exclude oxygen and moisture.

\section{Materials}

Commercial cyclohexane, diethylamine, and isoprene were purified by the usual methods. $n$-Butyllithium was prepared from $n$-butyl chloride and lithium metal in purified petroleum ether and was used as a cyclohexane solution.

Synthesis of 1-Diethylamino-3-methyl-2-butene (I)

To the cyclohexane $(50 \mathrm{~m} l)$ solution of isoprene $(6.8 \mathrm{~g}, 0.1 \mathrm{~mol})$, the amide-amine complex, prepared from $7.7 \mathrm{~g}(0.105 \mathrm{~mol})$ of diethylamine and $5 \mathrm{mmol}$ of $n$-butyllithium, was added. The mixture was heated at $50^{\circ} \mathrm{C}$ for $5 \mathrm{hr}$. Then a small amount of methanol was added in order to stop the reaction. After the solvent and other low boiling point materials were removed by evaporation, (I) was separated by fractional distillation in vacuo, bp $56^{\circ} \mathrm{C}(36 \mathrm{mmHg})$, yield $34 \%$.

Found: C, 76.81\%; H, 13.77\%; N, 9.91\%. Calcd for $\mathrm{C}_{9} \mathrm{H}_{19} \mathrm{~N}$ : C, $76.5 \% ; \mathrm{H}, 13.6 \% ; \mathrm{N}$, 9.9\%.

(I) gave reasonable IR and NMR spectra.

Procedures of the Rate Study

The methods of rate study of the addition reaction were described in a previous paper. ${ }^{3}$

\section{RESULTS AND DISCUSSION}

Selective Preparation of 1-Diethylamino-3-methyl2-butene (I)

As was reported previously, the addition reactions of lithium diethylamide onto butadiene and styrene did not take place in the absence of diethylamine. A similar phenomenon was also observed in the reaction of isoprene with lithium diethylamide. Under the synthetic conditions described in the experimental part, the sole product isolated was 1-diethylamino-3methyl-2-butene (I). The regioselectivity is es- timated to be more than $99 \%$ according to the vapor phase chromatographic analysis of the reaction product, though vpc did show a sign of the existence of a trace of some unidentified byproducts. The selective formation of (I) in the reaction of isoprene with $\mathrm{Et}_{2} \mathrm{NLi} \cdot 2 \mathrm{Et}_{2} \mathrm{NH}$ complex may be contrasted with the lower selectivity for the dimethylamino-olefin in the reaction with sodium dimethylamide in dimethylamine. ${ }^{11}$

\section{Kinetic Studies of the Addition Reaction}

The addition reaction of diethylamine onto isoprene catalyzed by lithium diethylamide is considered to proceed via the following equations. ${ }^{3}$

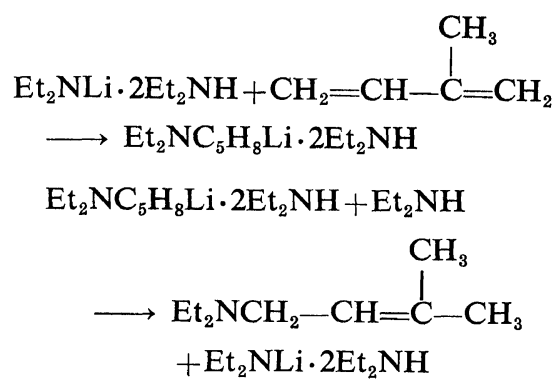

The rate of the addition reaction was found to change according to the first-order with respect to isoprene concentration, as shown in Figure 1. The pseudo first-order rate constant calculated from the results in Figure 1 is 0.27 $\mathrm{hr}^{-1}$. Dependency of the reaction rate on the concentration of lithium diethylamide was determined by analyzing the reaction rate under a constant concentration of isoprene, with the ratio of initial concentration of $\mathrm{Et}_{2} \mathrm{NH}$ to $\mathrm{Et}_{2} \mathrm{NLi}$ being 3.0.

$$
\left.v=k^{\prime} \text { [Isoprene }\right]
$$

where

$$
k^{\prime}=k_{\mathrm{I}}\left[\mathrm{Et}_{2} \mathrm{NLi}\right]^{x}
$$

The slope of the straight line obtained from the plot of $\log k^{\prime}$ vs. $\log \left[\mathrm{Et}_{2} \mathrm{NLi}\right]$ should show the dependency of the rate upon the lithium amide concentration. It is seen from Figure 2 that the reaction rate can be expressed as eq 5 since the slope of the line is 0.96 . The rate constant $k_{\mathrm{I}}$, calculated from eq 5 is $0.30 \times 10^{-3} l \mathrm{~mol}^{-1} \mathrm{sec}^{-1}$

$$
v=k_{\mathrm{I}}[\text { Isoprene }]\left[\mathrm{Et}_{2} \mathrm{NLi}\right]
$$


(in cyclohexane at $50^{\circ} \mathrm{C}$, with $\left[\mathrm{Et}_{2} \mathrm{NH}\right] /\left[\mathrm{Et}_{2} \mathrm{NLi}\right]=$ 3.0).

Since an isoprene molecule possesses only one reactive site that could accept the nucleophilic attack, instead of the two sites for a butadiene molecule, the value of the rate constant $k_{\mathrm{I}}$ observed for isoprene should be compared with that of $k_{\mathrm{B}} / 2$ for butadiene $\left(k_{\mathrm{B}}=0.85 \times 10^{-3}\right.$ $\left.l \mathrm{~mol}^{-1} \sec ^{-1}\right)$.

It is concluded from these data that the reactivity of isoprene is slightly smaller than that
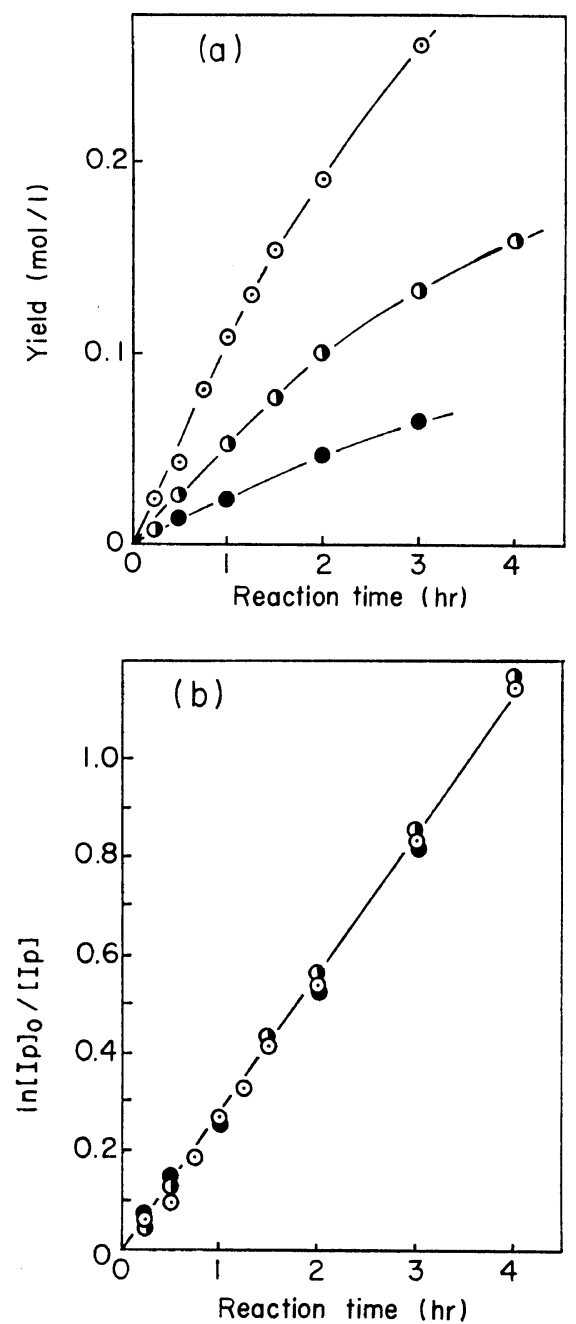

Figure 1. Time-conversion curves (a) and firstorder analysis (b) of the reaction of the $\mathrm{Et}_{2} \mathrm{NLi}-$ $\mathrm{Et}_{2} \mathrm{NH}$ complex with isoprene (Ip): $\left[\mathrm{Et}_{2} \mathrm{NH}\right]_{0} /$ $\left[\mathrm{Et}_{2} \mathrm{NLi}\right]_{0}, 3.0 ;\left[\mathrm{Et}_{2} \mathrm{NLi}\right]_{0}, 0.25 \mathrm{M}$; in cyclohexane at $50^{\circ} \mathrm{C}$; [Ip $]_{0}, 0.125 M(\bigcirc), 0.250 M(\odot), 0.500 M(\odot)$.

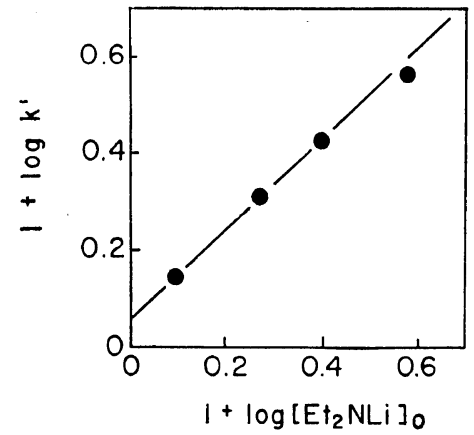

Figure 2. Relationship between lithium diethylamide concentration and pseudo first-order rate constant: $\left[\mathrm{Et}_{2} \mathrm{NH}\right]_{0} /\left[\mathrm{Et}_{2} \mathrm{NLi}\right]_{0}, \quad 3.0 ; \quad[\mathrm{Ip}]_{0}, \quad 0.25 \mathrm{M}$; in cyclohexane at $50^{\circ} \mathrm{C}$.

for butadiene. The comparison of the reactivity of isoprene with that of butadiene would be of interest in connection with the reactivity of these monomers in anionic polymerizations. In the addition reactions of butyllithium onto butadiene and isoprene, as was examined by $\operatorname{Sinn}^{7}$ for the initiation reactions, the rate constants for butadiene and isoprene were $4.5 \times 10^{-5}$ $l \mathrm{~mol}^{-1} \mathrm{sec}^{-1}$ and $1.6 \times 10^{-4} l \mathrm{~mol}^{-1} \mathrm{sec}^{-1}$, respectively; thus the reactivity of isoprene is about 7 times higher than that of butadiene. According to Sinn, the rate of the addition reaction would be controlled by the coordination step of diene monomers onto lithium species.

The slightly smaller reactivity of isoprene observed in the present study may suggest that the relative importance of the coordination step decreases to some extent with the amide-amine complex, probably owing to the polar nature of the N-Li bond compared to the $\mathrm{C}-\mathrm{Li}$ bond. The polar character of the amide-amine complex was previously demonstrated ${ }^{12}$ by the $\sigma-\rho$ Hammett plot of the rate of addition reaction onto $p$-substituted styrene derivatives, where the $\rho$-value was observed to be +5.0 ; this was almost the same value as that reported for the anionic polymerization of the styrene derivatives in the tetrahydrofuran.

Comparison with the Results of Tait, et al.

Tait and coworkers have recently reported ${ }^{4-6}$ that the polymerizations of styrene and isoprene initiated by lithium diethylamide itself take place in the polar solvents. The polymerization 
processes are anionic in nature and the polystyrene produced has $-\mathrm{NEt}_{2}$ as an end group of the polymer. The most remarkable feature of the polymerization is the sigmoidal timeconversion curves; the long induction period of a few hours is followed by fairly rapid propagation steps.

Contrary to these observations, our results showed that the addition reactions of lithium diethylamide onto dienes and styrene derivatives took place very rapidly from the early stage of the reactions in the presence of diethylamine, because lithium diethylamide was activated enough through the formation of one to two complexes with diethylamine, as reported previously. ${ }^{3}$ Furthermore, the diethylamine present in the reaction system promotes reaction (2) to produce again the one to two complexes, which can enter reaction (1) again, forming a reaction cycle between (1) and (2). As a consequence, the rate of consumption of diene or styrene should be much larger than that found in the polymerization reactions of these monomers with the use of lithium amide alone as the initiator.

\section{REFERENCES}

1. M. Szwarc, "Carbanions, Living Polymers and Electron Transfer Processes," Interscience Publishers, Inc., New York, N.Y., 1968.

2. N. Imai, T. Narita, and T. Tsuruta, Tetrahedron Lett., 38, 3517 (1971).

3. T. Narita, N. Imai, and T. Tsuruta, Bull. Chem. Soc. Jpn., 46, 1242 (1973).

4. A. C. Angood, S. A. Hurley, and P. J. T. Tait, J. Polym. Sci., Polym. Chem. Ed., 11, 2777 (1973).

5. A. C. Angood, S. A. Hurley, and P. J. T. Tait, ibid., Polym. Chem. Ed., 13, 2437 (1975).

6. S. A. Hurley and P. J. T. Tait, ibid., Polym. Chem. Ed., 14, 1565 (1976).

7. W. Gebert, J. Hinz, and H. Sinn, Makromol. Chem., 144, 97 (1971).

8. M. Morton, L. J. Fetters, R. A. Pett, and J. F. Meier, Macromolecules, 3, 327 (1970).

9. D. J. Worsfold and S. Bywater, ibid., 5, 393 (1972).

10. D. I. Hoke, D. L. Surbey, and W. R. Oviatt, J. Polym. Sci., Part A-1, 10, 595 (1972).

11. G. K. Noren, J. Org. Chem., 40, 967 (1975).

12. T. Narita, T. Yamaguchi, and T. Tsuruta, Bull. Chem. Soc. Jpn., 46, 3825 (1973). 